The Astrophysical Journal, 620:786-794, 2005 February 20

(C) 2005. The American Astronomical Society. All rights reserved. Printed in U.S.A.

\title{
QUIESCENT AND COHERENT CORES FROM GRAVOTURBULENT FRAGMENTATION
}

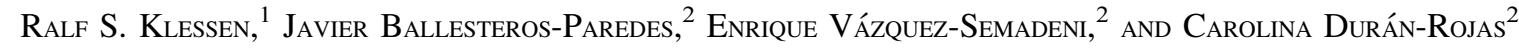 \\ Received 2003 June 2; accepted 2004 November 3
}

\begin{abstract}
We investigate the velocity structure of protostellar cores that result from nonmagnetic numerical models of the gravoturbulent fragmentation of molecular cloud material. A large fraction of the cores analyzed are "quiescent"; i.e., they have nonthermal line widths smaller or equal to the thermal line width. Specifically, about $23 \%$ of the cores have subsonic turbulent line-of-sight velocity dispersions $\sigma_{\text {turb }} \leq c_{s}$. A total of $46 \%$ are "transonic," with $c_{s}<\sigma_{\text {turb }} \leq 2 c_{s}$. More than half of our sample cores are identified as "coherent," i.e., with $\sigma_{\text {turb }}$ roughly independent of column density. Of these, about $40 \%$ are quiescent, $40 \%$ are transonic, and $20 \%$ are supersonic. The fact that dynamically evolving cores in highly supersonic turbulent flows can be quiescent may be understood because cores lie at the stagnation points of convergent turbulent flows, where compression is at a maximum and relative velocity differences are at a minimum. The apparent coherence may be due, at least in part, to an observational effect related to the length and concentration of the material contributing to the line. In our simulated cores, $\sigma_{\text {turb }}$ often has its local maximum at small but finite offsets from the column density maximum, suggesting that the core is the dense region behind a shock. Such a configuration is often found in observations of nearby molecular cloud cores and argues in favor of the gravoturbulent scenario of stellar birth as it is not expected in star formation models based on magnetic mediation. A comparison between the virial estimate $M_{\mathrm{vir}}$ for the mass of a core based on $\sigma_{\text {turb }}$ and its actual value $M$ shows that cores with collapsed objects tend to be near equipartition between their gravitational and kinetic energies, while cores without collapsed objects tend to be gravitationally unbound, suggesting that gravitational collapse occurs immediately after gravity becomes dominant. Finally, cores in simulations driven at large scales are more frequently quiescent and coherent and have more realistic ratios of $M_{\mathrm{vir}} / M$, supporting the notion that molecular cloud turbulence is driven at large scales.
\end{abstract}

Subject headings: ISM: clouds — ISM: kinematics and dynamics — stars: formation — turbulence

\section{INTRODUCTION}

Understanding the processes that lead to the formation of stars is one of the fundamental challenges in theoretical astrophysics. It is well known that stars form in dense cores within molecular clouds, but the physical processes that control the formation of low-mass stars within these cores are not well understood yet.

The traditional scenario assumes that low-mass protostellar cores are in quasi-static equilibrium supported against gravitational collapse by a combination of magnetic and thermal pressures (see, e.g., Shu et al. 1987). A core forms stars once magnetic support is lost through a process called ambipolar diffusion. Neutral gas particles slowly drift through the ions that are held up by the magnetic field, allowing the core to eventually attain a critical mass-to-flux ratio. Then the gravitational energy exceeds the magnetic energy, and collapse sets in from the inside out.

The theory of gravoturbulent star formation (see, e.g., the reviews by Vázquez-Semadeni et al. 2000; Larson 2003; Mac Low \& Klessen 2004 and references therein), on the other hand, suggests that clouds and cores are formed by compressible motions in the turbulent velocity field of their environment (e.g., von Weizsäcker 1951; Sasao 1973; Hunter \& Fleck 1982; Elmegreen 1993; Padoan 1995; Ballesteros-Paredes et al. 1999a, 1999b; Klessen et al. 2000; Padoan et al. 2001a; Heitsch

\footnotetext{
1 Astrophysikalisches Institut Potsdam, An der Sternwarte 16, 14482 Potsdam, Germany; rklessen@aip.de.

2 Centro de Radioastronomía y Astrofísica, UNAM, Apdo. Postal 72-3 (Xangari), Morelia, Michoacán 58089, Mexico; j.ballesteros@astrosmo.unam. mx, e.vazquez@astrosmo.unam.mx,c.duran@astrosmo.unam.mx.
}

et al. 2001; Vázquez-Semadeni et al. 2005). Those cores with an excess of gravitational energy collapse rapidly to form stars, while the others with sufficiently large internal or kinetic energies reexpand once the turbulent compression subsides.

Observational evidence suggests that low-mass stars form from molecular cloud cores with column density profiles that often resemble those of Bonnor-Ebert ${ }^{3}$ equilibrium spheres (Alves et al. 2001; see also the review by André et al. 2000) and with velocity dispersions that are small, i.e., transonic, or even subsonic (Myers 1983; Barranco \& Goodman 1998; Goodman et al. 1998; Jijina et al. 1999; Caselli et al. 2002; Tafalla et al. 2004). For this reason such cores are often termed "quiescent." Moreover, if the measured line-of-sight (LOS) velocity dispersion of a core is independent of column density toward the maximum, then it is called "coherent" (Barranco \& Goodman 1998). In the scenario of magnetically mediated star formation (Shu et al. 1987) these structures are explained as consequences of the quasi-static contraction process. In the gravoturbulent theory, however, protostellar cores are transient features naturally generated by the dynamical flow in the cloud. In order to test this theory, it is necessary to show that these fluctuations exhibit properties similar to those of the observed cores.

Several groups have now began to study core properties in numerical simulations of gravoturbulent cloud fragmentation (e.g., Ostriker et al. 2001; Padoan et al. 2001a, 2001b; BallesterosParedes \& Mac Low 2002; Gammie et al. 2003; Li et al. 2004; Schmeja \& Klessen 2004; Jappsen \& Klessen 2004; Tilley \&

\footnotetext{
3 Ebert (1955) and Bonnor (1956) describe the equilibrium density structure of isothermal gas spheres confined by an external pressure as a solution of the Lane-Emden equation.
} 
Pudritz 2004; Vázquez-Semadeni et al. 2005). In particular, Ballesteros-Paredes et al. (2003, hereafter Paper I) demonstrated that indeed transient, dynamic cores have an angle-averaged column density structure that often resembles that of hydrostatic Bonnor-Ebert profiles. This analysis was based on numerical calculations by Klessen et al. $(1998,2000)$ and Klessen \& Burkert $(2000,2001)$ and applied a fitting procedure similar to that used by Alves et al. (2001).

In this paper we focus on the velocity structure of protostellar cores and compare our model cores with the data available for observed quiescent, low-mass cores. In $\S 2$ we summarize the main features of the numerical models used and explain how we analyze the density and velocity structure. In $\S 3.1$ we show that the density fluctuations that we identify with protostellar cores often have very small and nearly spatially constant turbulent LOS velocity dispersion, even though they are produced by highly turbulent supersonic flows. In $\S 3.2$ we discuss the energy budget of the cores. Finally, in $\S 4$ we summarize and interpret our results in terms of the gravoturbulent fragmentation model of star formation.

\section{NUMERICAL SIMULATIONS AND CORE SAMPLE}

An important prerequisite for adequately describing the density and velocity structure of cores in numerical models of gravoturbulent molecular cloud evolution is the ability to resolve high-density contrasts at arbitrary locations within the cloud. Smoothed particle hydrodynamics (SPH; see Benz 1990; Monaghan 1992) is probably the best method currently available for this purpose.

The properties of our numerical scheme and resolution issues in the context of gravoturbulent fragmentation have been extensively discussed in Paper I (see also Klessen et al. 2000; Klessen 2001). Once the density contrast in the center of a collapsing cloud core exceeds a density contrast of about $10^{4}$, a "sink" particle is created (Bate et al. 1995). It replaces the central high-density region and has the ability to accrete further infalling material while keeping track of mass and linear and angular momentum. However, the internal structure of the sink particle is not resolved. With a diameter of about $600 \mathrm{AU}$ it fully encloses the star/disk system expected to form roughly $1000 \mathrm{yr}$ after the critical density for sink particle formation is reached (Wuchterl \& Klessen 2001).

The numerical resolution limit of our numerical scheme is determined by the Bate \& Burkert (1997) criterion, which is sufficient for the highly nonlinear fluctuation spectrum considered here. This is confirmed by resolutions studies with up to $10^{7} \mathrm{SPH}$ particles (see Jappsen et al. 2005). It should be noted, however, that the Bate \& Burkert (1997) criterion may not be sufficient for adequately following the growth of linear perturbations out of quasi-equilibrium, as was suggested for the case of rotationally supported disks by R. Fisher et al. (2004, private communication).

We analyze two models, one labeled LSD, in which turbulence is driven on large scales (of wavelength $\lambda \approx 1 / 2$ of the computational box), and the other labeled SSD, in which energy is injected on smaller scales (of $\lambda \approx 1 / 8$ of the box). We consider the system at an evolutionary stage when $5 \%$ of the available gas mass is accumulated in collapsed cores. Note that in Paper I we also studied cores from a contracting Gaussian density field (GC) without turbulence. Since in this paper we focus on the turbulent velocity structure, that simulation is not considered here.

To identify cloud cores, we use the three-dimensional clumpfinding algorithm introduced in Appendix A of Klessen \&
Burkert (2000). We then project a cubic subregion of the full computational volume centered around the core along the three principal axes and compute the column density $N$ and the total, turbulent-plus-thermal LOS velocity dispersion $\sigma_{\mathrm{LOS}}$ of each core. We take $\sigma_{\mathrm{LOS}}^{2}=\sigma_{\text {turb }}^{2}+c_{s}^{2}$, where the turbulent velocity dispersion $\sigma_{\text {turb }}$ is obtained as the mass-weighted standard deviation of the velocity field in each LOS along the projection axis, and $c_{s}^{2}$ is the sound speed of the mean particle $\left(\mu=2.3 m_{\mathrm{H}}\right)$. This assumes optically thin emission throughout the subcube and gives us a direct estimate of the true physical state of the system. A detailed comparison with observations requires one to consider specific molecular emission lines tracing various density regimes and to take optical thickness effects into account.

Since each projection in general gives different values of $N$, core size $R$, and $\sigma_{\mathrm{LOS}}$, we treat each projection as an independent case. We have increased the number of analyzed cores in comparison to Paper I by taking the first 200 cores identified in each of the LSD and SSD simulations. For the quiescence and coherence studies we only consider "starless" cores and exclude those with collapsed central regions, i.e., with a sink particle in their interior. For the energy budget analysis, however, we do include cores with sinks, as they allow for comparison with observations of cores containing young stellar objects. In order to avoid repetition, we analyze fields with multiple cores detected by the clump-finding algorithm only once. This procedure yields a sample of 44 cores for the LSD model and 101 for SSD without central collapsed object, plus 15 and 10 fields with sink particles for LSD and SSD models, respectively. Altogether, we analyze $(44+101) \times 3=435$ column density maps without, and $(15+10) \times 3=75$ column density maps with sink particles. Adopting the same physical scaling as in Paper I, the maps we consider cover 0.154 by $0.154 \mathrm{pc}$. The mean density in the simulation is $n\left(\mathrm{H}_{2}\right)=3.3 \times 10^{3} \mathrm{~cm}^{-3}$, the total mass in the simulation corresponds to $\sim 700 M_{\odot}$, and the speed of sound is $c_{s}=0.2 \mathrm{~km} \mathrm{~s}^{-1}$.

\section{RESULTS}

\subsection{Quiescent and Coherent Cores from Gravoturbulent Fragmentation}

In the following, we consider a core to be "quiescent" if its projected nonthermal velocity dispersion $\sigma_{\text {turb }}$ is smaller or equal to the thermal sound speed $\left(\sigma_{\text {turb }} \leq c_{s}\right)$ within the half central surface density contour, i.e., if the nonthermal component to the observed line width does not exceed the thermal line broadening. We call a core "coherent" if its velocity dispersion is independent of column density again for $N$ larger than half the peak value $N_{\max }$, or in other words, if $\sigma_{\text {turb }}$ is roughly independent of the offset from the core center.

In Figures 1 and 2 we illustrate the properties of four different molecular cloud cores in our models. We show maps of column density $N$ and maps of the LOS velocity dispersion $\sigma_{\text {LOS }}$ for each projection. To check for coherence, we also plot $\sigma_{\mathrm{LOS}}$ versus the normalized column density $N / N_{\max }$. In this particular case, we average $\sigma_{\mathrm{LOS}}$ in bins of 0.1 with respect to the normalized column density. This allows for a direct comparison, e.g., with Figure 4 in Barranco \& Goodman (1998), who plot the velocity dispersion against the antenna temperature using similar binning. The cores are chosen to span a wide range of morphological and structural appearance. Figure 1 shows two cores from model LSD. The first one is a smooth and roundish object that is coherent as well as quiescent in each of its projections. Its density as well as kinematic structure closely 

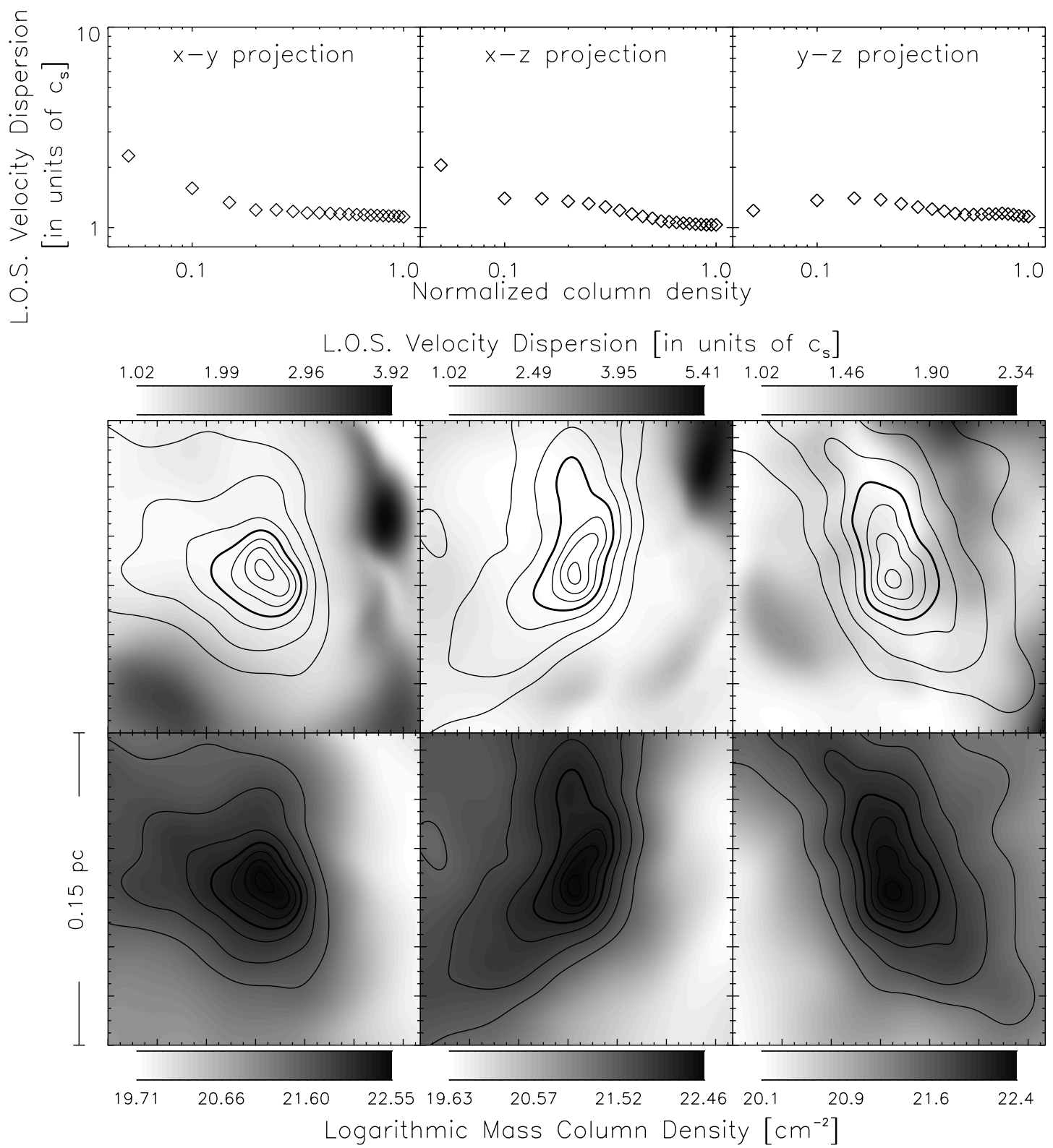

FIG. 1.-Two selected cores from model LSD in their three projections. For each core, the bottom panels give gray-scale maps of logarithmic column density $N$. The middle panels show the total, i.e., turbulent plus thermal, line-of-sight velocity dispersion $\sigma_{\mathrm{LOS}}=\left(\sigma_{\text {turb }}^{2}+c_{s}^{2}\right)^{1 / 2}$ superimposed on the column density contours. In the top panels we plot $\sigma_{\text {LOS }}$ against normalized column density $N$. We normalize $\sigma_{\text {LOS }}$ to the thermal sound speed $c_{s}$ as indicated by the upper gray-scale bar, and we plot $N$ in logarithmic units as indicated by the lower gray-scale bar. For better orientation, we also indicate the density structure with contour lines in the bottom and middle panels. Contour levels are drawn in linear scaling at $10 \%, 20 \%, 35 \%, 50 \%, 65 \%, 80 \%$, and $95 \%$ of the peak value $N_{\max }$. The $50 \%$ isocontour is marked with a thicker line.

resembles that of the two "thermal" cores L1498 and L1517B in Taurus, studied in great detail by Tafalla et al. (2004). In the inner parts the derived velocity dispersion is almost entirely determined by thermal motion only. The second core in Figure 1 has $x y$ and $x z$ projections that are classified as subsonic coherent, but it appears transonic coherent in the $y z$ map. Recall that, similarly to the standard observational procedures, we consider only column densities above half of the peak value. In contrast to the first core, it is clearly cometary shaped as a result of highly anisotropic ram pressure. The external flow coming from the right side (in the $x y$ and $x z$ projection) is abruptly stopped at the surface of the core, leading to a noticeable increase of the velocity dispersion there. In Figure 2 we present cores from model SSD. The first case is coherent only in the $y z$ map; in the other two projections $\sigma_{\text {LOS }}$ varies too strongly with location and con- sequently with $N$. The last one represents the subset of cores that are neither quiescent nor coherent in any of their projections.

The fact that high-density clumps in turbulent molecular clouds are created by convergent flows and thus are transiently bounded by ram pressure has observational consequences. As illustrated in Figures 1 and 2, in the simulations we often find localized maxima of $\sigma_{\mathrm{LOS}}$ in the low column density gas at the outskirts of the core, suggesting that the core is actually a dense postshock region, with the localized maximum of $\sigma_{\mathrm{LOS}}$ signaling the shock position. Such a configuration is also often seen in observations of actual molecular cloud cores (e.g., Barranco \& Goodman 1998; Goodman et al. 1998; Caselli et al. 2002; Tafalla et al. 2004). This important observational feature is thus naturally explained by the theory of gravoturbulent star formation. It should be noted that the "standard" scenario of 

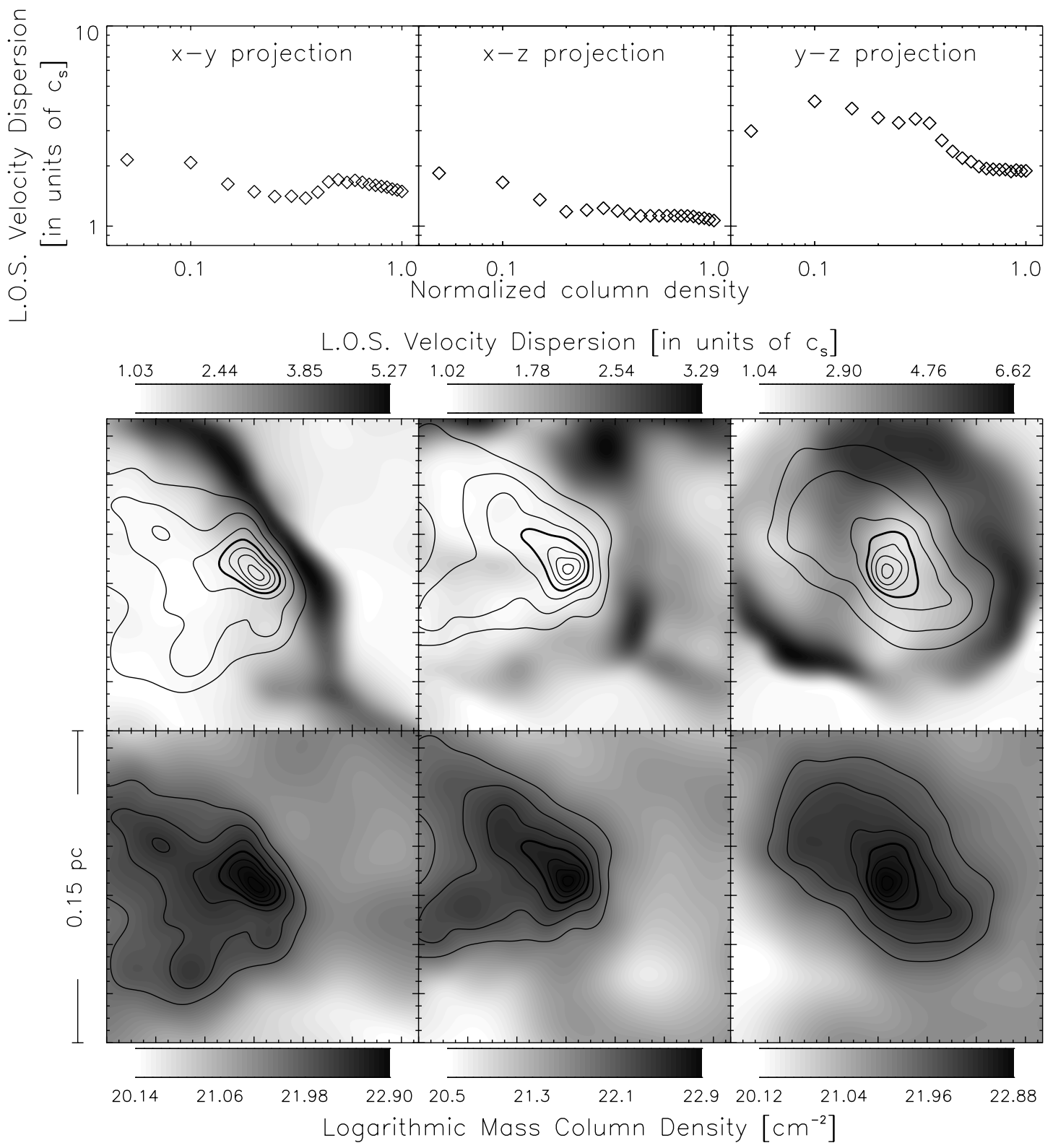

FIG. 1.-Continued

magnetically mediated star formation treats the turbulent nature of the cores' surroundings in a rather ad hoc fashion, through the consideration of microturbulent motions providing an isotropic pressure that increases with decreasing density (e.g., Lizano \& Shu 1989; Myers \& Fuller 1992). In this case, the increase of $\sigma_{\mathrm{LOS}}$ outside the core should in general be roughly isotropic, contrary to observed localized maxima of the velocity dispersion (see, e.g., maps in Barranco \& Goodman 1998; Goodman et al. 1998; Caselli et al. 2002).

The origin of the coherence, on the other hand, is not so clear. We speculate that it may arise from the fact that the densityweighted path length of gas contributing to the emission has a minimum at the core center, as illustrated in Figure 4 of Goodman et al. (1998). This contributing length is thus stationary with respect to small offsets in the plane of the sky. If the line width in a pencil beam is due essentially to densityweighted velocity differences sampled along the LOS, then it should exhibit the same behavior. Because our analysis is based on information from all the available gas, the quiescent and coherent nature of some cores in our sample is unequivocal evidence of the absence of large velocities in their interior. This suggests that either these cores have not developed gravitational collapse motions or are at the earliest stages of collapse, with velocities still being small. Whether this is the correct interpretation of observed line maps requires detailed radiative transfer calculations for various tracer molecules, and we plan to test this speculation in detail in a forthcoming paper.

From Figures 1 and 2 and the statistics of the complete core sample $^{4}$ (Table 1), we note several issues. First, as already discussed in Paper I, the inferred properties of cores may vary considerably between different projections. For example, $\sigma_{\text {turb }}$

${ }^{4}$ Note that choosing a different time for analysis in our simulations (cf. $\S 2$ ), or different parameters for the simulations, would probably alter to some extent the statistics presented in this paper. Thus, the precise fractions of coherent and quiescent cores should not be taken literally. The fundamental result is that substantial fraction of the cores in the simulations analyzed here, at an arbitrary time in their evolution, are quiescent and/or coherent. 

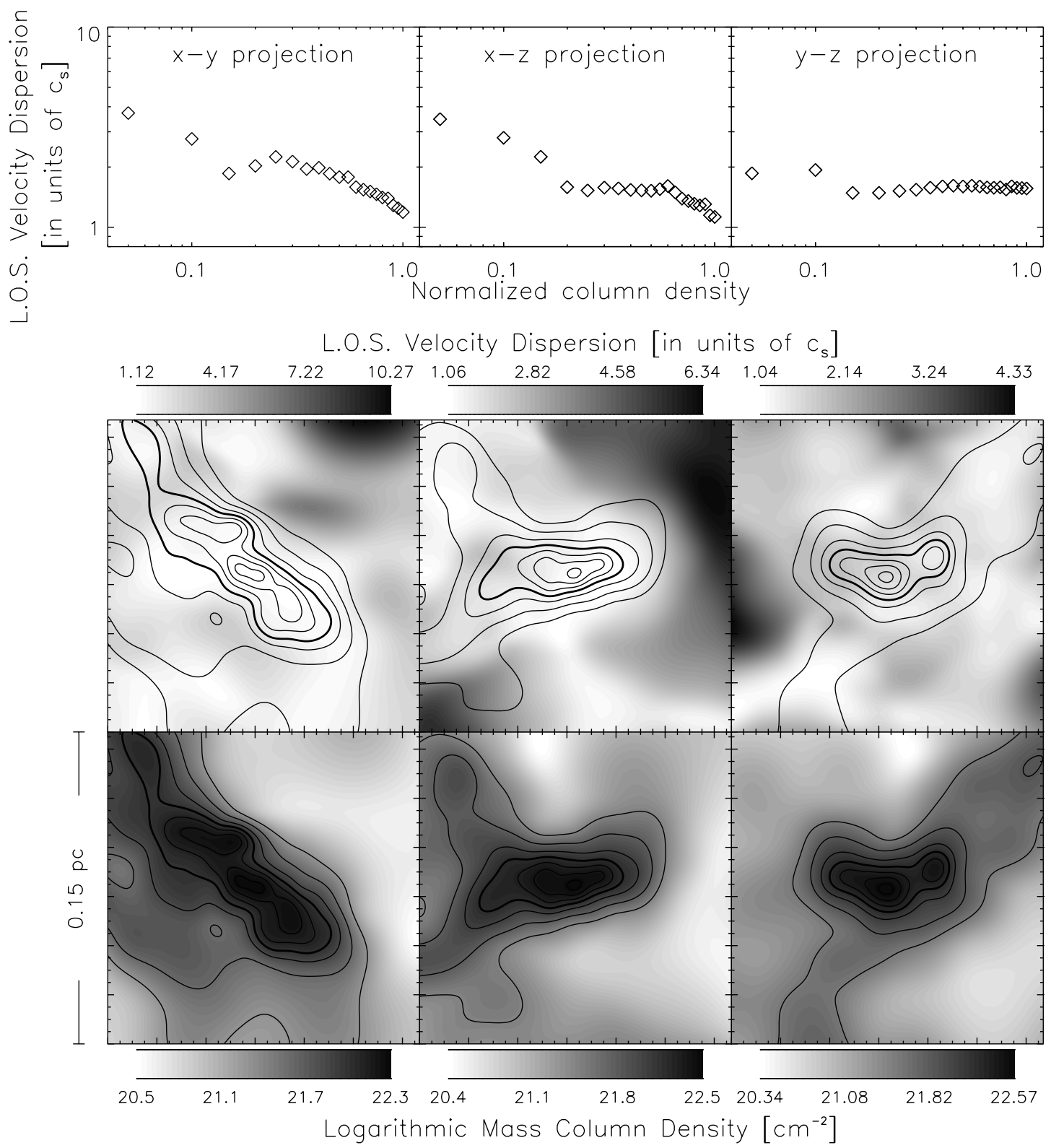

FIG. 2.-Two selected cores from SSD in their three projections. Notation and scaling is identical to Fig. 1.

may vary by as much as a factor of $2-3$. Second, in our turbulent models, roughly $60 \%$ of all cores can be identified as being coherent by visual inspection. About $40 \%$ of these are quiescent or subsonic, $40 \%$ are transonic, and about $20 \%$ are supersonic. Third, we note a clear distinction between the LSD and SSD models, the former having a larger fraction of coherent cores, most of which are also quiescent. Instead, the SSD model produces a larger number of cores that do not qualify as being coherent, and those that do are mostly transonic or supersonic. Thus, the LSD model compares better to observations than the SSD model, because observed cores often appear quiescent and coherent. However, persuasive observational statistics are still lacking. Altogether this argues in favor of clouds and their cores being driven from large scales (see also the discussion in Ossenkopf \& Mac Low 2002).

To estimate the fraction of cores with subsonic velocities, Figure 3 shows a histogram of the mean velocity dispersion inside the lowest contour (above half of the peak column density). For the whole core sample (models LSD and SSD combined), about $23 \%$ of all cores are quiescent in the strict sense (i.e., with $\sigma_{\text {turb }} \leq c_{s}$ ), while $46 \%$ are still transonic with $c_{s}<\sigma_{\text {turb }} \leq 2 c_{s}$. For the preferred model LSD, roughly $50 \%$ of them are coherent and subsonic, $15 \%$ are coherent and transonic, only $6 \%$ are coherent and supersonic, and the remaining 29\% are not found to be coherent.

We stress that all of this occurs in turbulent flows with rms Mach numbers as high as 6 . In fact, this is a natural consequence of the turbulent energy cascade. The velocity field becomes progressively more autocorrelated toward small scales, leading to the well-known line width-size relation in Galactic molecular clouds (Larson 1981). In interstellar turbulence, there is thus always a length scale at which the flow turns from supersonic to subsonic (Padoan 1995; Vázquez-Semadeni et al. 2003a). This does not necessarily imply that the dissipative regime of the turbulence has been reached, nor that there is a unique inner scale, but only that, on average, at this scale in a particular flow, the cascade turns into an incompressible one. In Galactic molecular clouds, this happens at roughly $0.05-0.1 \mathrm{pc}$ 

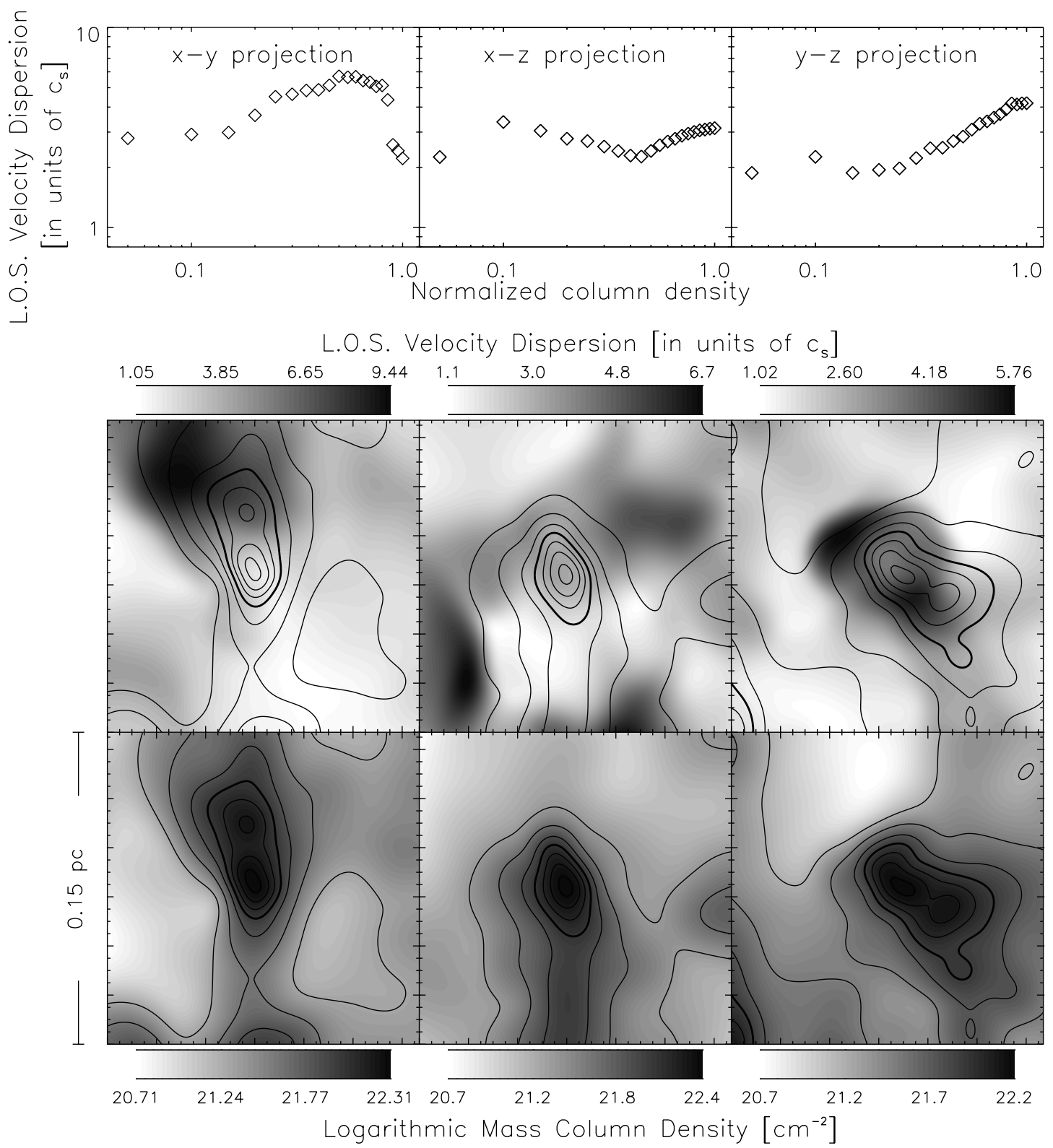

Fig. 2.-Continued

(e.g., Larson 1981; Myers 1983), which is the typical size of cold cloud cores. Again, this does not imply that these cores are quasi-static or long-lived. On the contrary, cores in our simulations are always out of equilibrium. They are created by a transient turbulent compression that eventually subsides, at which point the core is left in an unbalanced state. If the core crosses the "border" of gravitational instability, then it immediately proceeds to collapse. Otherwise, it reexpands and merges back

TABLE 1

Statistics of Coherent Cores

\begin{tabular}{cccc}
\hline \hline Type of Cores & Model LSD & Model SSD & Complete Sample \\
\hline Coherent subsonic........... & $51.5 \%$ & $12.9 \%$ & $24.6 \%$ \\
Coherent transonic .......... & $13.6 \%$ & $30.0 \%$ & $25.0 \%$ \\
Coherent supersonic........ & $6.0 \%$ & $13.5 \%$ & $11.3 \%$ \\
Incoherent.................... & $28.7 \%$ & $43.6 \%$ & $39.1 \%$ \\
Number of maps ............ & 132 & 303 & 435 \\
\hline
\end{tabular}

into its surroundings in times slightly longer than the local free-fall time (Vázquez-Semadeni et al. 2005). Moreover, cores can be disturbed, destroyed or recompressed by interaction with neighboring turbulent fluctuations (e.g., Klessen et al. 2000; Klessen 2001).

\subsection{Energy Balance in Protostellar Cores}

Observed protostellar cores are often said to be in "virial equilibrium" (e.g., Myers 1983; Myers \& Goodman 1988a, 1988b), because the measured and the inferred "virial" values of certain physical variables are comparable. Recent studies have focused on the comparison between the observationally estimated mass $M$ and the virial mass $M_{\mathrm{vir}}$, defined as

$$
M_{\mathrm{vir}} \equiv 210[R /(1 \mathrm{pc})]\left[\Delta v_{\text {eff }} /\left(1 \mathrm{~km} \mathrm{~s}^{-1}\right)\right]^{2} M_{\odot}
$$

(see, e.g., Caselli et al. 2002; Tachihara et al. 2002) for a uniform density sphere of radius $R$ and "effective" line width $\Delta v_{\text {eff }}$. Because most observations are based on the emission from tracer 


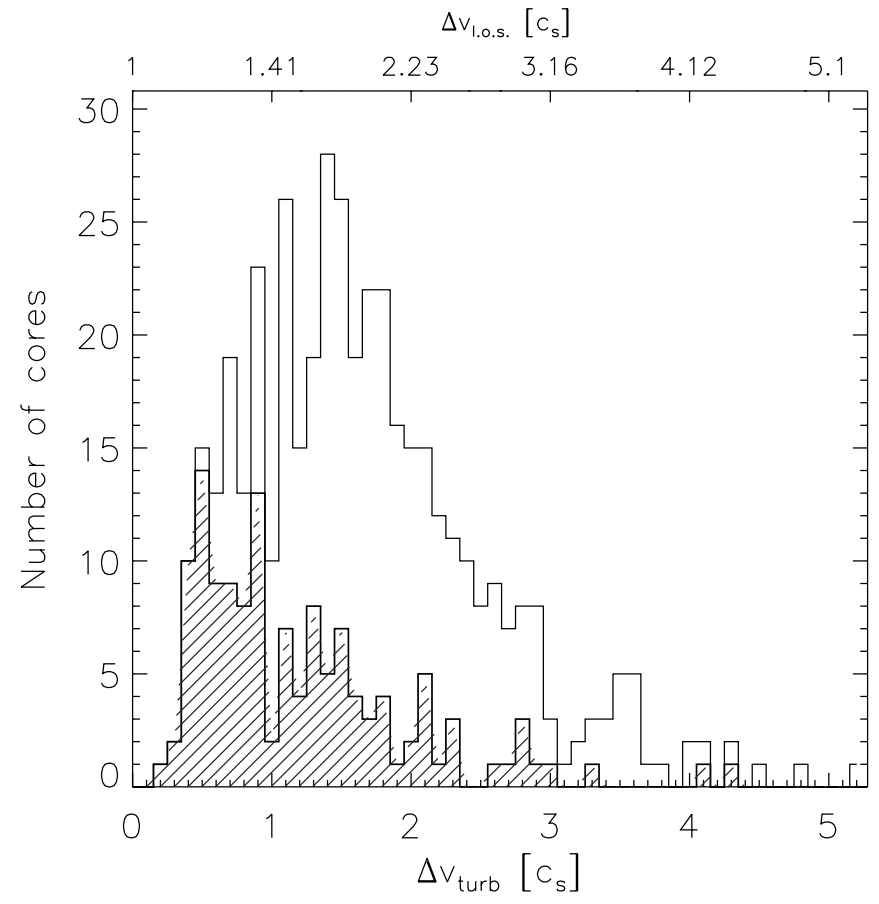

FIG. 3.-Histogram of the mean LOS velocity dispersion $\sigma_{\text {LOS }}$ inside half of the maximum column density. Bottom abscissa gives the scaling for the turbulent LOS velocity dispersion $\sigma_{\text {turb }}$, and the top abscissa denotes total LOS velocity dispersion $\sigma_{\text {LOS }}$, given by $\left(\sigma_{\text {turb }}^{2}+c_{s}^{2}\right)^{1 / 2}$. The distribution in model LSD is given by the hatched histogram; the histogram of model SSD is open.

molecules heavier than the mean particle mass in the gas ( $\left.\mu=2.3 m_{\mathrm{H}}\right)$, the thermal contribution to $\Delta v_{\text {eff }}$ must be calculated by subtracting the one from the tracer molecule and adding a fictitious contribution from the mean molecule (see, e.g., eq. [7] of Caselli et al. 2002). Given the definition of the LOS velocity dispersion $\sigma_{\operatorname{LOS}}$ in $\S 2$, for our theoretical data this "effective" line width is computed as $\Delta v_{\text {eff }}=(8 \ln 2)^{1 / 2} \sigma_{\text {LOS }}$.

In Figure 4 we plot $M_{\text {vir }}$ against $M$. The actual core mass $M$ is calculated by integrating the column density within the halfmaximum isocontour of the column density maps. The diagonal line denotes equipartition, $M_{\mathrm{vir}}=M$. Crosses (triangles) denote cores in the SSD (LSD) model. Cores with protostars (i.e., in our numerical scheme with a sink particle in their center) are indicated by tailed squares. Note that their virial mass estimates $M_{\mathrm{vir}}$ are lower limits. Although the sink particle carries the correct mass, there is no information on its internal velocity structure, because it is not resolved in the SPH code. Thus, the velocity dispersion calculated in fields with sink particles is an underestimate. However, this underestimate does not appear to be too serious, since at the evolutionary stage we consider, the mass in the central protostar is just a small fraction of the overall core mass. Moreover, a similar underestimate is likely to occur in the observations due to depletion and optical thickness effects in the dense, collapsing gas in the central regions of real cores. To allow for direct comparison with observational data, we also indicate in Figure 4 the regions covered by the cores in the surveys of Morata et al. (2004; vertical lines), Onishi et al. (2002; horizontal lines), Caselli et al. (2002; lines tilted by $-45^{\circ}$ ), and starless cores in Tachihara et al. (2002; lines tilted by $\left.+45^{\circ}\right)$.

With the above considerations in mind, several points are worth noting. First, cores from the large-scale turbulence model LSD populate a different region in $M_{\mathrm{vir}}-M$ parameter space than their counterparts in the small-scale turbulence model SSD. The

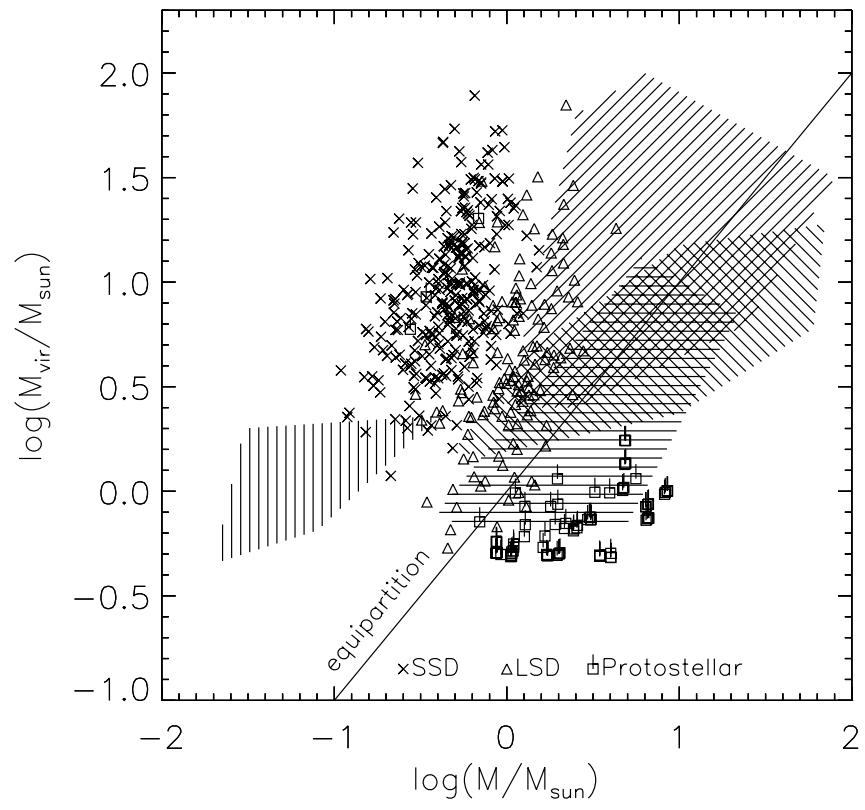

FIG. 4.-Estimated virial mass $M_{\text {vir }}$ plotted against actual mass $M$ for all analyzed cores in the simulations. Crosses denote cores in the small-scale turbulence model (SSD) and triangles denote cores in the large-scale turbulence model (LSD). Tailed squares indicate the lower limits on the estimates on $M_{\text {vir }}$ for cores in LSD with protostellar objects (sink particles) in their interior. Note that all three projections for each core are plotted independently. The identity $M_{\mathrm{vir}}=M$ is given by the solid line. We also indicate the parameter space covered by cores in the observational surveys by Morata et al. (2005) with vertical lines, by Onishi et al. (2002) with horizontal lines, by Caselli et al. (2002) with $-45^{\circ}$ lines, and we plot the starless cores in Tachihara et al. (2002) with $+45^{\circ}$ lines.

former tend to have somewhat lower LOS velocity dispersion $\sigma_{\text {LOS }}$ (implying lower virial masses) and at the same time larger actual masses. As a result, they are closer to equipartition than cores in SSD. The velocity field in the LSD model is dominated by large-scale shocks that are very efficient in sweeping up molecular cloud material, thus creating massive coherent density structures that more frequently exceed the critical mass for gravitational collapse. Therefore, cloud cores in the LSD model predominantly form in a clustered and coeval mode from gas that is largely Jeans unstable (see Klessen 2001). On average they have higher density contrast than their SSD counterparts, and their energy budget is more influenced by self-gravity. The LSD cores fall almost completely in the low-mass regions of the Onishi et al. (2002), Caselli et al. (2002), and Tachihara et al. (2002) samples, suggesting that the model reproduces well the physics of the lower mass cores in these regions, while the production of higher or lower mass cores by the simulations probably requires a different normalization and/or varying the mass content in the simulation (by varying the number of Jeans masses in the box).

On the other hand, shock-generated clumps in the SSD model tend to form at random locations and at random times. On average they have a smaller density contrast and smaller masses. By the same token, their velocity dispersions are larger, thus giving larger virial masses $M_{\text {vir }}$. These cores tend to be more dominated by their internal velocity dispersion rather than by self-gravity, as reflected by the fact that they exhibit larger departures from equipartition (typically by factors of 10-100), which appear inconsistent with observational estimates of this balance, except for the strongly subvirial cores presented by Morata et al. (2004). 
Second, the starless cores in our sample exhibit an excess of their virial mass. This result is consistent with recent observations by Tachihara et al. (2002) and Morata et al. (2004), who show that for cores without a central protostar the virial mass estimates usually exceed the actual mass. Although in the Tachihara et al. (2002) sample departures from equipartition are smaller (less than a factor of 10), Morata et al. (2004) show cores that depart from equipartition by a factor of 30 . On the other hand, the cores classified as starless by Caselli et al. (2002) tend to be in equipartition. These results suggest that some of the cores may actually contain collapsed objects. Observational evidence of this possibility has been given recently by new observations with the Sptizer Space Telescope. On one hand, Reach et al. (2004) have detected eight embedded sources (Class 0/I) in a small field centered on a single globule in $\operatorname{Tr} 37$ in which only one IRAS source was detected. Similarly, Young et al. (2004) show that L1014, a dense core previously thought to be starless, actually shows evidence of containing an embedded source. Thus, if this phenomenon is common, then many apparently starless cores that are near equipartition may actually contain collapsed objects. Furthermore, as pointed out by Young et al. (2004), this would also suggest that traditional estimates of prestellar core lifetimes may be overestimated. This again supports the idea that not all density peaks in self-gravitating turbulent fields necessarily collapse but that collapse occurs rapidly once equipartition is reached (Vázquez-Semadeni et al. 2005).

A third point to make is that cores with central protostars (sink particles) in our models tend to be more massive than cores without, in agreement with the observational situation that cores with stars tend to be more massive than starless cores (Caselli et al. 2002; Tachihara et al. 2002).

Finally, we note that even though some cores in the model lie close to the identity line $M_{\text {vir }}=M$, it does not imply virial equilibrium. This requires the second derivative of the moment of inertia to vanish (see, e.g., Ballesteros-Paredes 2004 and references therein). Our cores are not static but instead are constantly evolving, and thus they are in general out of equilibrium. Reaching hydrostatic equilibrium in a turbulent molecular cloud environment is extremely difficult and requires strongly idealized conditions that are not met in the interstellar gas. The condition $M_{\text {vir }}=M$, or equivalently $\sigma_{\mathrm{LOS}} \approx \sigma_{\text {vir }}$, simply reflects equipartition between the volume-averaged kinetic energy and self-gravity, as occurs precisely at the verge of gravitational collapse.

Together, our calculations support the following evolutionary sequence. Initially, cloud cores are generated by transient compressive turbulent motions. In this phase their energy budget is dominated by the external ram pressure. The compression causes their internal and gravitational energies to increase. If they accumulate enough mass or reach sufficient density contrast, they can become gravitationally unstable and quickly go into collapse. The transition to the stage when self-gravity dominates the evolution is characterized by approximate energy equipartition. If self-gravity never becomes that important, the cores are left with an excess of internal energy after the external compression subsides and can reexpand within a few free-fall times.

\section{SUMMARY AND CONCLUSIONS}

In the emerging picture of gravoturbulent star formation (see, e.g., the review by Mac Low \& Klessen 2004 and references therein), the structure of Galactic molecular clouds is determined by compressible supersonic turbulence. High-density cores build up at the stagnation points of locally convergent flows. Some of those cores may become gravitationally unstable and go into collapse to form stars, while others will simply redisperse into the ambient medium (Sasao 1973; Hunter \& Fleck 1982; Elmegreen 1993; Ballesteros-Paredes et al. 1999a; Padoan et al. 2001a; Klessen et al. 2000; Padoan \& Nordlund 2002; Vázquez-Semadeni et al. 2005).

Our analysis demonstrates that a considerable fraction of the cores in supersonic turbulent flows can be identified as being "quiescent" (i.e., sub- or transonic) and "coherent" (i.e., with roughly constant velocity dispersion across the central parts of the core) despite the fact that they are embedded and formed in a highly dynamical environment. These cores are quiescent, because they form at the stagnation points of the flow at which the compression is at a maximum and the relative velocity differences are at a minimum. The origin of coherence is not so clear, but we speculate that it may be caused by projection, because the LOS length of the matter contributing to the line profile has a minimum at the core center, and small offsets from the center therefore cause little variation in the observed line width.

Molecular cloud cores that harbor protostars in their interior (as identified by the presence of sink particles in our models) are characterized by having $M_{\mathrm{vir}}<M$, but for those objects our estimates for $M_{\text {vir }}$ are lower limits to the true value. On the other hand, most of the cores without central objects in our simulations have $M_{\mathrm{vir}}>M$ and thus are not gravitationally bound. This is in agreement with the observational results of Tachihara et al. (2002) and Morata et al. (2005) for starless cores. However, some observed "starless" cores also seem to lie close to or even fall below the equipartition line (e.g., Caselli et al. 2002), and we speculate that at least some of them may contain as yet undetected young stellar or substellar objects, as in the case of core L1014 (Young et al. 2004).

The fact that cores in the gravoturbulent model are initially created and confined by the ram pressure from convergent larger scale flows leads to the velocity dispersion being highest in the low column density gas at the surface of the clump, either at localized positions or with bowlike shapes. Such structure is often found in detailed high-resolution velocity maps of observed starless cores (e.g., Barranco \& Goodman 1998; Goodman et al. 1998; Caselli et al. 2002; Tafalla et al. 2004). However, it is not predicted or explained within the framework of star formation models based on the slow contraction of magnetically subcritical cores mediated by ambipolar diffusion.

Our calculations support an evolutionary sequence in which a molecular cloud core is formed by turbulent ram pressure compression. As it gains mass and becomes denser, both its internal kinetic energy and the absolute value of its gravitational energy increase. For some cores, the gravitational attraction may exceed any opposing forces (either thermal or magnetic), and the core goes into collapse to quickly build up a protostellar object in its interior. The fact that star-forming cores are often observed near energy equipartition is only the signature of gravity becoming dynamically important, not of hydrostatic equilibrium. However, if the external turbulent compression ends before the core reaches the state where it is dominated by self-gravity, then it will reexpand and merge with the lower density ambient molecular cloud material.

In a typical turbulent cloud environment, the evolution of molecular cloud cores is both transient and fast. This holds for their formation by convergent flows, as well as for their destruction either by collapse and transformation into stars, or by reexpansion or dispersion by passing shock fronts. Both observational 
estimates of prestellar core lifetimes in well characterized starforming regions (e.g., Lee \& Myers 1999; Jijina et al. 1999) and numerical simulations of star-forming turbulent clouds (e.g., Vázquez-Semadeni et al. 2005) indicate that the process of core formation and collapse is fast $\left(\$ 10^{6} \mathrm{yr}\right) .^{5}$

\footnotetext{
${ }^{5}$ Note that it is often thought that transient cores cannot form stars, while star-forming cores are the ones "that last long enough" to do so. For example, Tassis \& Mouschovias (2004) suggest that typical molecular cloud cores undergo a lengthy gestation period before becoming observable and being able to form stars. In their picture, starless cores are simply not old enough yet to begin forming stars. They thus conclude that observational estimates of core lifetimes do not constrain the duration of such lengthy early phase. However, this picture has been challenged. Observationally, the existence of such a long gestation period would imply that most molecular clouds should appear devoid of star formation, in contradiction with observational facts (Hartmann 2003; BallesterosParedes \& Hartmann 2005). Theoretically, the long gestation period can only occur in a quiescent, unperturbed medium. The presence of supersonic turbulence speeds up the formation and evolution of the density fluctuations (i.e., of the cores; see Li \& Nakamura 2004; Vázquez-Semadeni et al. 2005), independently of whether they end up collapsing or redispersing.
}

We conclude that the quiescent, coherent, and sometimes near-virial nature of observed molecular cloud cores is in direct agreement with the theory of gravoturbulent star formation (Mac Low \& Klessen 2004 and references therein), in which stars build up from material in the gravitationally unstable parts of the spectrum of transient, dynamically evolving density fluctuations that are the characteristics of self-gravitating, supersonically turbulent media such as interstellar gas clouds.

We thank L. Hartmann, S. Lizano, and P., Myers for stimulating discussions during the elaboration of the present manuscript. We also thank our referee A. Goodman for insightful comments and suggestions. R. S. K. acknowledges support by the Emmy Noether Program of the Deutsche Forschungsgemeinschaft (DFG: grant KL1358/1). J. B. P. and E. V. S. acknowledge support from CONACYT's grants 27752-E and 36571-E, respectively. This research has made use of NASA's Astrophysics Data System Abstract Service.

\section{REFERENCES}

Alves, J., Lada, C. J., \& Lada, E. A. 2001, Nature, 409, 159

André, P., Ward-Thompson, D., \& Barsony, M. 2000, in Protostars and Planets IV, ed. V. Mannings, A. Boss, \& S. Russell (Tucson: Univ. Arizona Press), 59

Ballesteros-Paredes, J. 2004, Ap\&SS, 289, 243

Ballesteros-Paredes, J., \& Hartmann, L. 2005, ApJ, submitted

Ballesteros-Paredes, J., Hartmann, L., \& Vázquez-Semadeni, E. 1999b, ApJ, 527,285

Ballesteros-Paredes, J., Klessen, R. S., \& Vázquez-Semadeni, E. 2003, ApJ, 592, 188 (Paper I)

Ballesteros-Paredes, J., \& Mac Low, M. 2002, ApJ, 570, 734

Ballesteros-Paredes, J., Vázquez-Semadeni, E., \& Scalo, J. 1999a, ApJ, 515, 286

Barranco, J. A., \& Goodman, A. A. 1998, ApJ, 504, 207

Bate, M. R., Bonell, I. A., \& Price, N. M. 1995, MNRAS, 277, 362

Bate, M. R., \& Burkert, A. 1997, MNRAS, 288, 1060

Benz, W. 1990, in Proc. of the NATO Advanced Research Workshop on The Numerical Modeling of Nonlinear Stellar Pulsations, ed. J. R. Buchler (Dordrecht: Kluwer), 269

Bonnor, W. B. 1956, MNRAS, 116, 351

Caselli, P., Benson, P. J., Myers, P. C., \& Tafalla, M. 2002, ApJ, 572, 238

Ebert, R. 1955, Z. Astrophys., 36, 222

Elmegreen, B. G. 1993, ApJ, 419, L29

Gammie, C. F., Lin, Y., Stone, J. M., \& Ostriker, E. C. 2003, ApJ, 592, 203

Goodman, A. A., Barranco, J. A., Wilner, D. J., \& Heyer, M. H. 1998, ApJ, 504,223

Hartmann, L. 2003, ApJ, 585, 398

Heitsch, F., Mac Low, M.-M., \& Klessen, R. S. 2001, ApJ, 547, 280

Hunter, J. H., \& Fleck, R. C. 1982, ApJ, 256, 505

Jappsen, A.-K., \& Klessen, R. S. 2004, A\&A, 423, 1

Jappsen, A.-K., Klessen, R. S., Larson, R., Li, Y., \& Mac Low, M.-M. 2005, A\&A, in press (astro-ph/0410351)

Jijina, J., Myers, P. C., \& Adams, F. C. 1999, ApJS, 125, 161

Klessen, R. S. 2001, ApJ, 556, 837

Klessen, R. S., \& Burkert, A. 2000, ApJS, 128, 287

$$
\text { 2001, ApJ, 549, } 386
$$

Klessen, R. S., Burkert, A., \& Bate, M. R. 1998, ApJ, 501, L205

Klessen, R. S., Heitsch, F., \& Mac Low, M.-M. 2000, ApJ, 535, 887

Larson, R. B. 1981, MNRAS, 194, 809

2003, Rep. Prog. Phys., 66, 1651
Lee, C. W., \& Myers, P. C. 1999, ApJS, 123, 233

Li, P. S., Norman, M. L., Mac Low, M.-M., \& Heitsch, F. 2004, ApJ, 605, 800 Li, Z.-Y., \& Nakamura, F. 2004, ApJ, 609, L83

Lizano, S., \& Shu, F. H. 1989, ApJ, 342, 834

Mac Low, M.-M., \& Klessen, R. S. 2004, Rev. Mod. Phys., 76, 125

Monaghan, J. J. 1992, ARA\&A, 30, 543

Morata, O., Girart, J. M., \& Estalella, R. 2005, A\&A, in press (astro-ph/ 0412621)

Myers, P. C. 1983, ApJ, 270, 105

Myers, P. C., \& Fuller, G. A. 1992, ApJ, 396, 631

Myers, P. C., \& Goodman, A. A. 1988a, ApJ, 326, L27 1988b, ApJ, 329, 392

Onishi, T., Mizuni, A., Kawamura, A., Tachihara, K., \& Fukui, Y. 2002, ApJ, 575,950

Ossenkopf, V., \& Mac Low, M.-M. 2002, A\&A, 390, 307

Ostriker, E. C., Stone, J. M., \& Gammie, C. F. 2001, ApJ, 546, 980

Padoan, P. 1995, MNRAS, 277, 377

Padoan, P., Goodman, A., Draine, B. T., Juvela, M., Nordlund, Å., \& Rögnvaldsson, Ö. E. 2001b, ApJ, 559, 1005

Padoan, P., Juvela, M., Goodman, A. A., \& Nordlund, Å. 2001a, ApJ, 553, 227

Padoan, P., \& Nordlund, Å. 2002, ApJ, 576, 870

Reach, W. T., et al. 2004, ApJS, 154, 385

Sasao, T. 1973, PASJ, 25, 1

Schmeja, S., \& Klessen, R. S. 2004, A\&A, 419, 405

Shu, F. H., Adams, F. C., \& Lizano, S. 1987, ARA\&A, 25, 23

Tachihara, K., Onishi, T., Mizuno, A., \& Fukui, Y. 2002, A\&A, 385, 909

Tafalla, M., Myers, P. C., Caselli, P., \& Walmsley, C. M. 2004, A\&A, 416, 191

Tassis, K., \& Mouschovias, T. Ch. 2004, ApJ, 616, 283

Tilley, D. A., \& Pudritz, R. E. 2004, MNRAS, 353, 769

Vázquez-Semadeni, E., Ballesteros-Paredes, J., \& Klessen, R. S. 2003a, ApJ, $585, \mathrm{~L} 131$

Vázquez-Semadeni, E., Kim, J., Shadmehri, M., \& Ballesteros-Paredes, J. 2005, ApJ, 618, 344

Vázquez-Semadeni, E., Ostriker, E. C., Passot, T., Gammie, C., \& Stone, J. 2000, in Protostars \& Planets IV, ed. V. Mannings, A. Boss, \& S. Russell (Tucson: Univ. Arizona Press), 3

von Weizsäcker, C. F. 1951, ApJ, 114, 165

Wuchterl, G., \& Klessen, R. S. 2001, ApJ, 560, L185

Young, C. H., et al. 2004, ApJS, 154, 396 